\title{
Article \\ Coupling and Optical Analysis of a Round-Cornered Square-Shaped Microresonator
}

\author{
Prabodh Panindre ${ }^{1,+}$, N.S.Susan Mousavi ${ }^{2, *}++^{\mathbb{D}}$, Bruna Paredes ${ }^{3} \mathbb{D}$, Mahmoud Rasras $^{3}$ and Sunil Kumar $^{1,3}(\mathbb{D}$ \\ 1 Mechanical Engineering Department, New York University Tandon School of Engineering, \\ 6 Metrotech Center, Brooklyn, NY 11201, USA; prabodh@nyu.edu (P.P.); sunil.kumar@nyu.edu (S.K.) \\ 2 Applied Physics Research Laboratory (APRL), School of Physics, Institute for Research in Fundamental \\ Sciences IPM, Tehran 9538-33511, Iran \\ 3 Engineering Division, New York University Abu Dhabi, Saadiyat Island, Abu Dhabi P.O. Box 129188, \\ United Arab Emirates; bp64@nyu.edu (B.P.); mr5098@nyu.edu (M.R.) \\ * Correspondence: susan.mousavi@nyu.edu or s.mousavi@ipm.ir \\ + These authors contributed equally to this work.
}

Citation: Panindre, P.; Mousavi, N.S.S.; Paredes, B.; Rasras, M.; Kumar, S. Coupling and Optical Analysis of a Round-Cornered Square-Shaped Microresonator. Appl. Sci. 2021, 11, 8659. https://doi.org/10.3390/ app11188659

Academic Editor: Michael (Misha) Sumetsky

Received: 19 August 2021

Accepted: 14 September 2021

Published: 17 September 2021

Publisher's Note: MDPI stays neutral with regard to jurisdictional claims in published maps and institutional affiliations.

Copyright: (c) 2021 by the authors. Licensee MDPI, Basel, Switzerland. This article is an open access article distributed under the terms and conditions of the Creative Commons Attribution (CC BY) license (https:// creativecommons.org/licenses/by/ $4.0 /)$.

\begin{abstract}
An on-chip structure consisting of a round-cornered square-shaped (RCSS) resonator as an optical filter is studied via optical experiments, analytical modeling, and numerical techniques. A general coupling model is shown to accurately represent the entire spectral response; the model also provides an understanding of the influence of geometrical and coupling parameters on the resonance characteristics of the RCSS microresonators. The selection of an optimum radius of curvature for rounding off the sharp corners of square microresonators can provide higher quality factors than that of conventional circular resonators. The rotation of the RCSS at the central corner coupling point is also shown to improve the quality factor and remove the minimal phase mismatch requirement and dependency on interaction length. The model results are validated with an electromagnetic finite domain analysis (EMFD) and optical experiments, for which an RCSS on a silicon-on-insulator platform is fabricated. It is shown that the optical performance characteristics (quality factor, transmission ratio, and extinction ratio) of the microresonators are very sensitive to the coupling parameters and must be carefully considered in addition to geometrical length, rotation, and curvature effects. Due to the change in coupling introduced by rotation, the quality factor of the round-cornered square-shaped microresonator can be significantly larger than a circular ring with the same size, thereby establishing RCSS as a competitive alternative to circular microresonators.
\end{abstract}

Keywords: microring resonator; fiber waveguides; couplers; round-cornered square-shaped microring; on-chip structure; propagation; scattering; losses

\section{Introduction}

Optical filters and their various types, including ring resonators with high-performance parameters, are among the advanced optical devices that have been used in both industrial and medical applications such as optical components, wavelength division multiplexing (WDM) devices, sensing platforms, among others [1-8]. The two main categories of optical components introduced in the literature are the free-space configurations (appropriate for off-chip devices) and the waveguide-type configurations (appropriate for on-chip devices). In both categories, the filtering mechanism is based on the modulation of the optical characteristics of the incident beam as well as the refractive index (RI) of the layers in slab-guiding structures, resulting in the selective reflection of a narrow band from a wide, specified spectral range and its transmission on both the short and long wavelength sides of this band.

The topmost desired characteristics of optical filters are their tunability, high-quality factor, a low full-width at half-maximum (FWHM) resonance, and small size. To improve the performance of the optical filters, several measures have been implemented. Recently, 
by manipulating fabrication procedures to reduce scattering, absorption, and intrinsic losses, researchers have demonstrated an ultra-sensitive resonator with a quality factor of up to 422 million [9]. In another study, the coupling loss was lowered by tailoring the directional coupler excitation to the resonator, which experimentally achieved a quality factor of 81 million on the $S_{3} N_{4}$ optical resonator platform [10]. Brunetti et al. [11] studied a $1 \mathrm{D}$ photonic crystal ring resonator and demonstrated a quality factor of more than $10^{10}$ at $1.55 \mu \mathrm{m}$.

In this realm, ring resonators and their variants such as racetrack configurations have good performance parameters and find various applications such as in optical networks and as sensors, for which they have been extensively studied in theory and for practical applications [8,12-17]. For racetrack resonators, it was found that the radius of curvature and the length of the coupling region play an essential role in the total optical loss, where a larger radius of curvature reduces the bending loss while increasing the scattering loss. In order to minimize overall loss and improve the quality factor, optimal values for the radius of curvature and the length of the straight coupling section were obtained [18]. However, concerns about short interaction lengths between the circular portion of such resonators and the straight waveguides $[19,20]$ have led researchers to consider polygonal shapes [21,22]. In the absence of sharp corners, the mode changes adiabatically between the radial mode in the curved sections and the normal mode in the straight section $[19,23]$.

In order to address the issues above and to obtain high optical length interaction, a lot of efforts have been made, including the proposition of various structures as well as the use of novel materials. As an example, polygonal microresonators have been introduced due to their ability to provide long interaction lengths and ease the air-gap spacing tolerance [24-26]. A longer interaction length and an optimal gap can create a strong modal field overlap of the resonator and the waveguide, which is essential for a strong coupling. However, it was found through a systematic study that the long interaction length between the waveguide and the resonator may make the coupling strength very sensitive to any phase mismatch between the waveguide mode and that of the resonator [20]. On the other hand, the application of polygonal optical microresonators with sharp corners can be limited by severe energy loss at the corners. Panindre et al. [27] incorporated a rounded fillet design into the square resonator, allowing the electromagnetic wave to slowly turn from one side of the square to the other side, thus reducing the optical loss at the sharp corners. When the radius of curvature was varied while maintaining a constant optical path length, they showed that for the single-mode square-shaped microresonators, the quality factors could be significantly increased. At an optimum radius of curvature, it was seen that the quality factor could even be one order higher than that of conventional circular microring resonators. However, the quality factor was found to be lower for several other values of radii of curvature, for which a few possible reasons were provided and discussed [27,28].

In the present work, an optical filter with a round-cornered square-shaped (RCSS) microring resonator is considered and its performance is analyzed. To benchmark the structural and optical characteristics of an RCSS, a coupled-mode theory is employed in the present study and compared to electromagnetic finite domain (EMFD) analysis and experiments.

Previously, the differences in optical losses in the resonator due to the variation of geometric parameters such as the length of straight stretches, the radius of curvature, and coupling length were conjectured to be the main cause of the changes in the optical response; however, the present results indicate that the nature and characteristics of the coupling are also major drivers of the variations. The effects of the radius of curvature and the RCSS rotation on the performance of the filter are numerically investigated and experimentally validated. 


\section{Coupling Model and Analysis}

In 1969, Marcatili [29] presented one of the preliminary coupling models for an integrated optical ring resonator. Since then, waveguide-resonator coupling has been studied for various types of geometries [11,12,30-32]. Based on the coupled-mode theory, a mathematical model was developed to analyze the effect of curvature on waveguide modes and to simulate the transmission spectra of the 1D photonic crystal ring resonator [11]. Using the scattering theory, Yariv and co-workers modeled the coupling and showed that the reflection and transmission properties of the waveguide are primarily influenced by the waveguide-resonator coupling, symmetry and degeneracy of the resonant modes, mode resonant frequency, and cavity-loss [12,30]. Several researchers were able to achieve optimum quality factor and extinction ratio [31,32] just by tuning the coupling parameters between the resonator and the input waveguide.

In the present study, a simple form of an optical filter with a round-cornered squareshaped (RCSS) resonator is used and shown in Figure 1. The optical source from the left-hand side of the structure is coupled with the bus waveguide and excites the electromagnetic wave in the RCSS resonator with a narrow-band resonance, leading to the light filtering. A sharp dip exists in the resonance transmission spectra ; hence, a good quality filtering wavelength is obtained in the transmission spectrum.

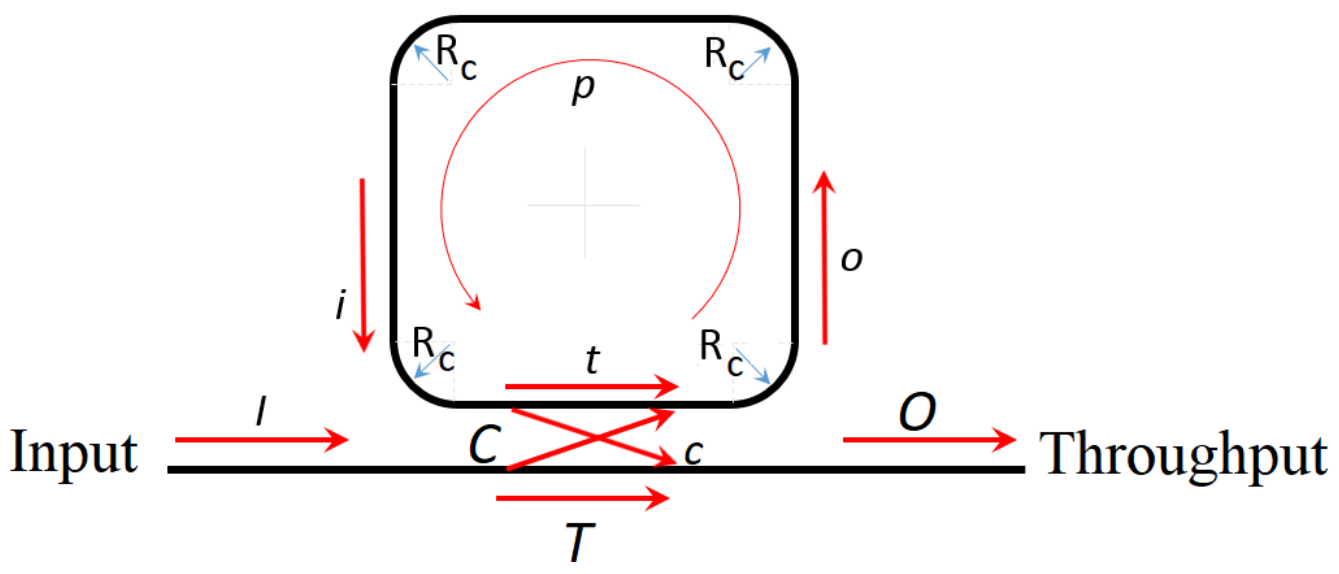

Figure 1. Schematic of a planar waveguide-coupled RCSS microresonator channel.

The complex output fields $O$ and $o$ are related to the complex input fields $I$ and $i$ through the self-coupling and cross-coupling coefficients $T$ and $t$, and $C$ and $c$, respectively. The optical coupling can be expressed as follows [33]:

$$
\left[\begin{array}{l}
O \\
o
\end{array}\right]=\left[\begin{array}{ll}
T & C \\
C & t
\end{array}\right]\left[\begin{array}{l}
I \\
i
\end{array}\right]
$$

All the variables in Equation (1) are complex values. Since $o$ is coupled back with the input $i$, we have:

$$
i=p o
$$

where $p$ is the propagation along the resonator. From the equation above, we obtain the following:

$$
\frac{O}{I}=\frac{T-(T t-C c) p}{1-t p}
$$

Tracking the energy in the waveguide and the ring resonator gives:

$$
\begin{aligned}
& |O|^{2}=|T I|^{2}+|c i|^{2} \\
& |o|^{2}=|C I|^{2}+|t i|^{2}
\end{aligned}
$$


or

$$
|O|^{2}+|o|^{2}=\underbrace{\left(|T|^{2}+|C|^{2}\right)}_{L^{2}}|I|^{2}+\underbrace{\left(|c|^{2}+|t|^{2}\right)}_{l^{2}}|i|^{2}
$$

where $L^{2}$ and $l^{2}$ are the loss variables of the waveguide and the resonator, respectively.

The left side of Equation (5) can also be expanded by using Equation (1), and using complex conjugates to compute absolute values yields the following:

$$
\left.\begin{array}{l}
T c^{*}+C t^{*}=0 \\
T^{*} c+C^{*} t=0
\end{array}\right\} \rightarrow \frac{|T|^{2}}{|t|^{2}}=\frac{|C|^{2}}{|c|^{2}}
$$

where the variables with an asterisk $*$ denote complex conjugates.

With the help of the set of equations in Equation (6), Equation (3) can be rewritten as:

$$
\frac{O}{I}=\frac{t^{*}-l^{2} p}{1-t p} \frac{T}{t^{*}}
$$

To simplify the result further, the phases $\phi_{p}$ and $\phi_{t}$ are introduced as:

$$
\begin{aligned}
& p=\bar{p} e^{j \phi_{p}} \\
& t=\bar{t} e^{j \phi_{t}}
\end{aligned}
$$

where $\bar{p}$ and $\bar{t}$ are magnitudes of $p$ and $t$, respectively. By defining the scaled resonator propagation value and the coupling region transmission value as $\alpha=\bar{p} l$ and $\beta=\bar{t} / l$, respectively, we get:

$$
\frac{O}{I}=\frac{\beta-\alpha e^{j \phi}}{1-\alpha \beta e^{j \phi}} \frac{T}{\beta}
$$

where $\phi=\phi_{t}+\phi_{p}$.

The energy transmission ratio (transmissivity) to throughput port $\tau$ using Equation (9) can be expressed as follows:

$$
\tau=\left|\frac{O}{I}\right|^{2}=\frac{O O^{*}}{I I^{*}}=\frac{\alpha^{2}+\beta^{2}-2 \alpha \beta \cos \phi}{1+\alpha^{2} \beta^{2}-2 \alpha \beta \cos \phi}\left|\frac{T}{\beta}\right|^{2}
$$

In a coupling condition when resonance occurs, minimum energy is transmitted through the throughput port, that is:

$$
\tau_{\min }=\left.\tau\right|_{\phi=0}=\frac{\alpha^{2}+\beta^{2}-2 \alpha \beta}{1+\alpha^{2} \beta^{2}-2 \alpha \beta}\left|\frac{T}{\beta}\right|^{2}=\frac{(\alpha-\beta)^{2}}{(1-\alpha \beta)^{2}}\left|\frac{T}{\beta}\right|^{2}
$$

Similarly, the energy transmitted to the throughput port is maximum when:

$$
\tau_{\max }=\left.\tau\right|_{\phi=\pi}=\frac{\alpha^{2}+\beta^{2}+2 \alpha \beta}{1+\alpha^{2} \beta^{2}+2 \alpha \beta}\left|\frac{T}{\beta}\right|^{2}=\frac{(\alpha+\beta)^{2}}{(1+\alpha \beta)^{2}}\left|\frac{T}{\beta}\right|^{2}
$$

The value of the $\tau_{\text {min }}$ becomes zero if $\alpha=\beta$, that is:

$$
\bar{p} l=\frac{\bar{t}}{l} \rightarrow \bar{p} l^{2}=\bar{t}
$$

If $\alpha=\beta=1, \tau_{\text {min }}$ would become undefined, as seen in Equation (11). However, this would yield the following:

$$
\bar{p} l=\frac{\bar{t}}{l}=1 \rightarrow \bar{t}=l, \quad \bar{p}=\frac{1}{l}
$$


Since $\bar{t}^{2}+\bar{c}^{2}=l^{2}$, we would get $\bar{c}^{2}=0$ if $\beta=1$, which means that there would be no coupling. Thus, $\alpha=\beta=1$ is not physically possible.

The transmission at full-width at half-maximum (FWHM) in this configuration is given by:

$$
\tau_{\text {FWHM }}=\tau_{\max }-\frac{\tau_{\max }-\tau_{\min }}{2}=\frac{\tau_{\max }+\tau_{\min }}{2}
$$

Substituting the $\tau_{\min }$ and $\tau_{\max }$ from Equations (11) and (12) into Equation (15), we get:

$$
\tau_{F W H M}=\frac{1}{2}\left(\frac{(\alpha+\beta)^{2}}{(1+\alpha \beta)^{2}}+\frac{(\alpha-\beta)^{2}}{(1-\alpha \beta)^{2}}\right)\left|\frac{T}{\beta}\right|^{2}
$$

$\tau_{\text {FWHM }}$ can also be found in Equation (10):

$$
\tau_{F W H M}=\frac{\alpha^{2}+\beta^{2}-2 \alpha \beta \cos \phi_{F W H M}}{1+\alpha^{2} \beta^{2}-2 \alpha \beta \cos \phi_{F W H M}}\left|\frac{T}{\beta}\right|^{2}
$$

Comparing Equations (16) and (17) and simplifying the expression, $\cos \phi_{F W H M}$ is expressed as:

$$
\cos \phi_{F W H M}=\frac{2 \alpha \beta}{1+\alpha^{2} \beta^{2}}=\frac{2 \bar{t} \bar{p}}{1+\bar{t}^{2} \bar{p}^{2}}
$$

Defining $\gamma$ as the ratio of the $\Delta \lambda_{F W H M}$ (spectral full-width at half-maximum, which is the wavelength spacing at the half level of the resonance intensity peak) to the $\Delta \lambda_{F S R}$ (the free spectral range or the wavelength spacing between two successive optical intensity maxima or minima), together with the help of Equation (18), we have the following:

$$
\gamma=\frac{\Delta \lambda_{F W H M}}{\Delta \lambda_{F S R}}=\mathcal{F}^{-1}=\frac{2 \phi_{F W H M}}{2 \pi}=\frac{1}{\pi} \cos ^{-1}\left(\frac{2 \alpha \beta}{1+\alpha^{2} \beta^{2}}\right)
$$

where $\mathcal{F}$ is the finesse, a quality measure of the microring, which is the ratio between the FSR and the FWHM. High finesse devices have a small FWHM, and a strong intensity build-up in the ring when in resonance is thus a measure of the sharpness of resonances relative to their spacing [15,34]. For simplicity, we introduce $X=\alpha \beta$ and $\epsilon=\tau_{\min } / \tau_{\max }$.

$$
X=\alpha \beta=\frac{\cos (\pi \gamma)}{1+\sin (\pi \gamma)}
$$

Substituting $\tau_{\min }$ and $\tau_{\max }$ from Equations (11) and (12) yields:

$$
\epsilon=\frac{\tau_{\min }}{\tau_{\max }}=\left[\frac{(\beta-\alpha)(1+\alpha \beta)}{(\beta+\alpha)(1-\alpha \beta)}\right]^{2}=\left[\frac{\left(X-\alpha^{2}\right)(1+X)}{\left(X+\alpha^{2}\right)(1-X)}\right]^{2}
$$

The extinction ratio $r_{e}$ can be computed from the equation above, where $r_{e}=10$ $\log \left(\tau_{\min } / \tau_{\max }\right)=10 \log (\epsilon)$ [31,32]. For an expression for $\alpha$, Equation (21) is rearranged and simplified as follows:

$$
\alpha^{2}=X \frac{[(1+X) \pm \sqrt{\epsilon}(1-X)]^{2}}{[(1+X)+\sqrt{\epsilon}(1-X)][(1+X)-\sqrt{\epsilon}(1-X)]}
$$

therefore,

$$
\alpha^{2}=X A \text { and } \beta^{2}=\frac{X}{A}
$$

where,

$$
A=\frac{[(1+X)+\sqrt{\epsilon}(1-X)]}{[(1+X)-\sqrt{\epsilon}(1-X)]}
$$


From the experimental or numerical simulation results, the steps to compute $\alpha$ and $\beta$ are as follows. By noting the observed full-width at half-maximum (FWHM) values, $X(=\alpha \beta)$ can be obtained by using Equations (19) and (20). Looking at the observed transmission values at the throughput port, $\epsilon$ can be found using Equation (21). From the $X$ and $\epsilon$ values, $A$ can be computed from Equation (24), which gives $\alpha$ and $\beta$ for the resonator-waveguide assembly.

\section{Optical Experimental Setup}

The designed optical rings are fabricated on a silicon-on-insulator platform with a $2 \mu \mathrm{m}$ thick cladding box and a $220 \mathrm{~nm}$ thick silicon layer, using the NanoSOI process available at Applied Nanotools Inc, NRC-NANO Building, 11,421 Saskatchewan Drive NW, Edmonton, Alberta T6G 2M9 Canada [35]. This process is based on direct-write $100 \mathrm{keV}$ electron beam lithography technology (EBL), in which hydrogen silsesquioxane (HSQ) resists and an anisotropic ICP-RIE etch process with chlorine is used. Through the plasma-enhanced chemical vapor deposition (PECVD) process, and with tetraethyl orthosilicate (TEOS) at $300^{\circ} \mathrm{C}$ as base, a $1 \mu \mathrm{m}$ oxide cladding was deposited on top. As a final step, $150 \times 150 \mu \mathrm{m}$ oxide windows were etched away to expose each of the devices to air. A scanning electron microscopy (SEM) image of the fabricated RCSS is shown in Figure 2.

The experimental setup is built with a tunable Keysight 81600B laser source, emitting light in the O-band combined with an electronic polarizer, which is used to select and maintain TE and TM polarizations. Single-mode lensed fibers are used to butt-couple the light into the SiPh chip and to collect it at the throughput port of the device. In order to improve the coupling efficiency, edge strip couplers, tapered from $18 \mathrm{~nm}$ to the desired waveguide width, are designed at the input and output of the chip. At the end, light is detected using a Keysight N7744A optical detector sensor, which can be read through the Keysight N77x viewer software. The schematic figure of the setup is seen in Figure 3.

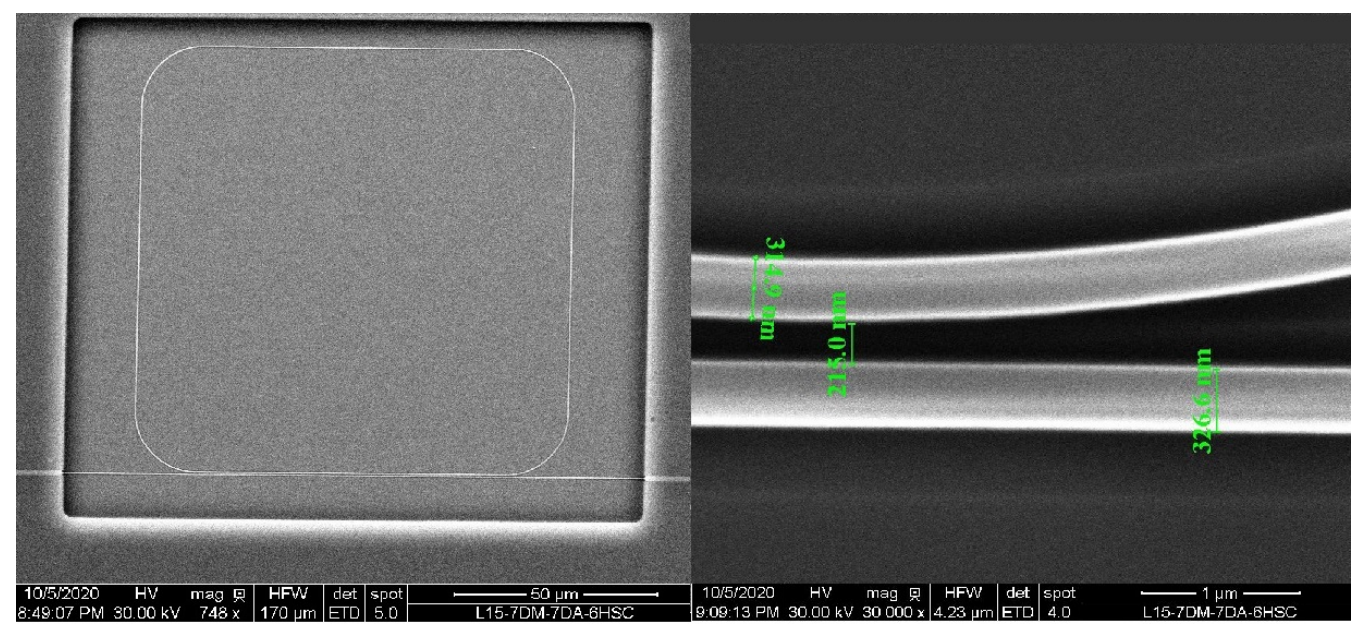

Figure 2. Scanning electron microscopy (SEM) image of the fabricated RCSS $\left(R_{c}=15 \mu \mathrm{m}\right)$ microresonator for optical experiments. 


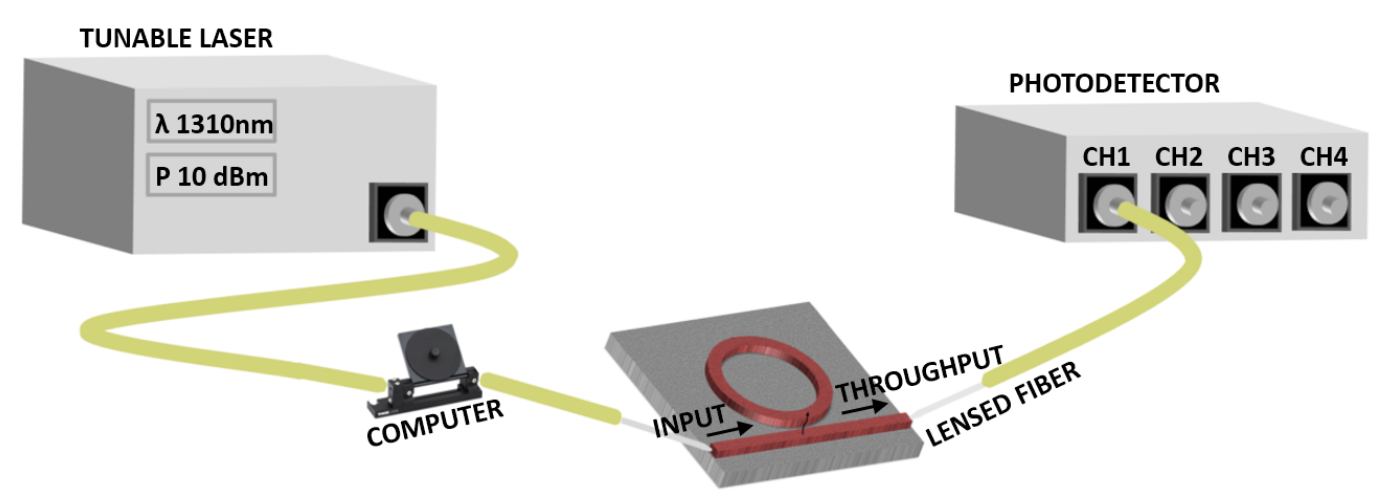

Figure 3. Schematic configuration of the experimental setup.

For the characterization of the optical transmission response, the light coupled with the device is optimized for the TE and TM polarizations, from an input power of $10 \mathrm{dBm}$ set at the laser source. The data are collected using the Keysight insertion loss software, which sweeps from $1309 \mathrm{~nm}$ to $1311 \mathrm{~nm}$ wavelengths in order to obtain the $\mathrm{O}$ band responses. The step size used for the wavelength was $0.2 \mathrm{pm}$.

\section{Results and Discussion}

The performance of the RCSS structures are evaluated by screening several parameters, including finesse and quality factor; the results are discussed below. Note that the quality factor $Q$ provides an absolute measure for the wavelength selectivity of the microring resonator. The quality factor and finesse are related as follows:

$$
Q=\frac{\lambda_{0}}{\Delta \lambda_{F W H M}}=\frac{\lambda_{0}}{\Delta \lambda_{F S R}} \frac{\Delta \lambda_{F S R}}{\Delta \lambda_{F W H M}}=\frac{\lambda_{0}}{\Delta \lambda_{F S R}} \mathcal{F}
$$

\subsection{Effects of Propagation ( $\alpha$ ) and Transmission ( $\beta$ )}

The effects of coupling transmission on the transmission characteristics of the RCSS in order to find an optimal set of parameters are investigated. $\beta$ has been increased from 0.6 to 1 , while other parameters are kept unchanged. A general parametric response that illustrates the wavelength spectra near resonance is presented in Figure 4 for different values of the resonator propagation value $\alpha$, and the coupling region transmission value $\beta$. As the resonator becomes lossier (i.e., the value of resonator propagation $\alpha$ reduces), the resonance peak becomes broader and the quality factor decreases. Losses in the RCSS resonators have previously been identified to be a result of (i) radial radiation losses at the bends, (ii) the transition losses in the regions where bends and straight segments connect, and (iii) the intrinsic propagation losses per unit length along with the entire travel distance in the microresonator [27].

From Figure 4, it can be seen that in addition to the intrinsic losses in the resonator above, the characteristics of the coupling also significantly influence the spectral response of the coupled waveguide-resonator system. The dip in the transmissivity at the throughput port at resonance is a function of the difference between $\alpha$ and $\beta$ and is maximized, i.e., the transmissivity ratio $\epsilon$ is at a minimum $(=0)$ when the values of $\alpha$ and $\beta$ are the same (see Equations (11) and (12)). Thus, as shown in Figure 5, to get the maximum spectral transmissivity dip (minimum $\epsilon$ ) at the throughput port at resonance, the intrinsic propagation property $\alpha$ of the resonator should be closest to the coupling transmission $\beta$, which is a function of the design of the coupling region. 


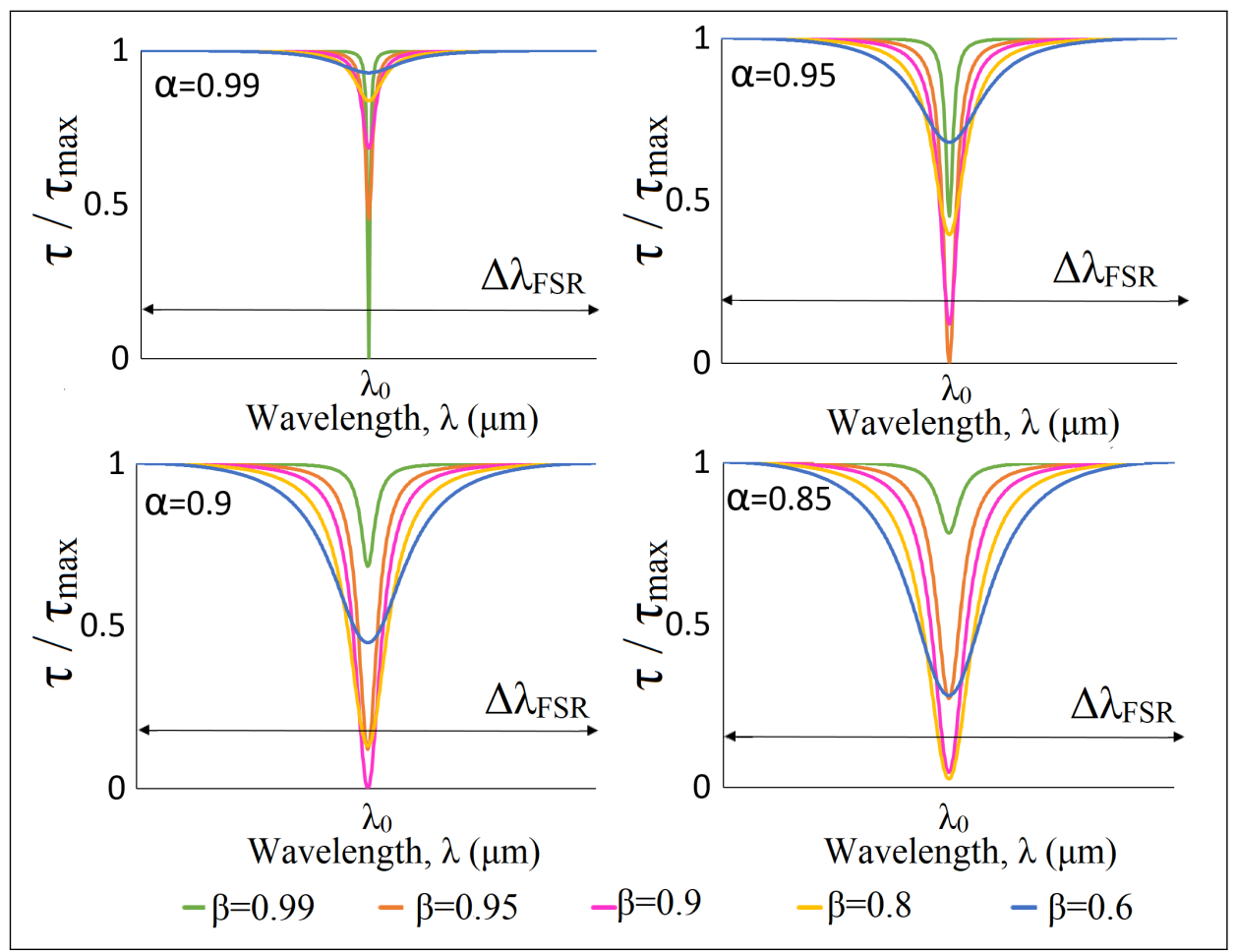

Figure 4. Effect of transmission $(\beta)$ and propagation $(\alpha)$ on optical response around the resonant wavelength $\left(\lambda_{0}\right)$.

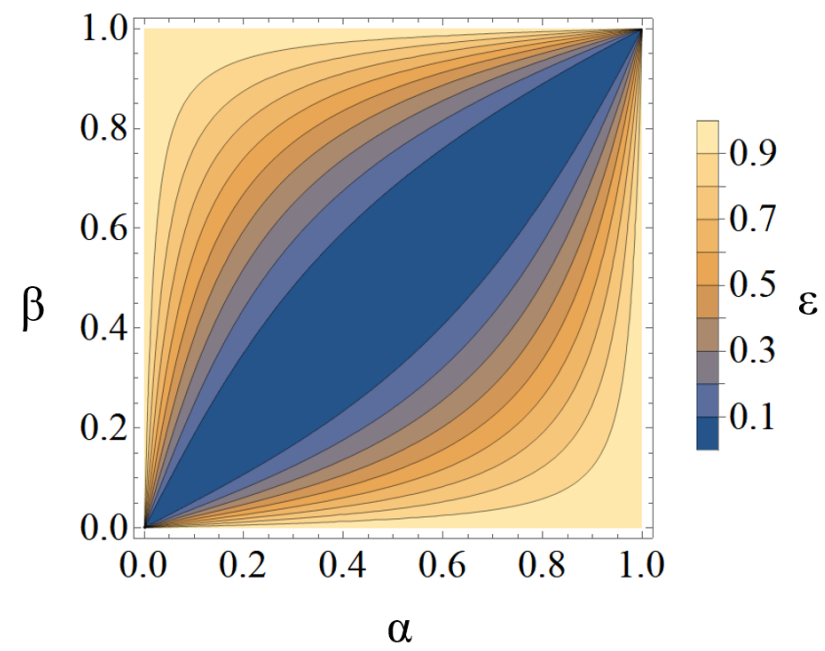

Figure 5. Variation of the transmission ratio $\epsilon$ (Equation (21)) at the resonant wavelength $\left(\lambda_{0}\right)$ as propagation $(\alpha)$ and transmission $(\beta)$ parameters change for an RCSS microresonator; $\epsilon=0$ (ideal) when $\alpha=\beta$.

For a given propagation value $\alpha$, higher values of the coupling transmission $\beta$ yield narrower resonance peaks and therefore higher quality factors. This phenomenon is captured in Figure 6 where the finesse $\mathcal{F}$ is shown to span several orders of magnitude, which is the same trend for the quality factor $Q$ as per Equation (25). The quality factor is a function of the product of $\alpha$ and $\beta$, which is unlike the dependency of throughput port transmissivity on the difference of $\alpha$ and $\beta$. The quality factor $Q$ increases monotonically as a function of $\alpha \beta$. Thus, to obtain the largest throughput port transmissivity dip, i.e., the lowest $\tau / \tau_{\max }$ and the highest extinction ratio, accompanied by the highest quality factor $Q$, the values of $\alpha$ and $\beta$ should be close to unity and as close to each other as possible. This would correspond to a lossless or near-lossless resonator, with a coupling design 
that gives the highest coupling transmission. Note that the higher the transmission $\beta$, the lower the coupling $c$ is. However, the value of $\beta$ can never be unity since that would imply $c=0$, which is only theoretically possible for an isolated microresonator unaffected optically by the waveguide, i.e., not coupled with the waveguide (see Equation (14)). An isolated lossless microresonator would theoretically possess a flat spectral response, except for a delta-function change at resonance, and it is the design of the coupling and its characteristics that introduce the broadened spectral response.

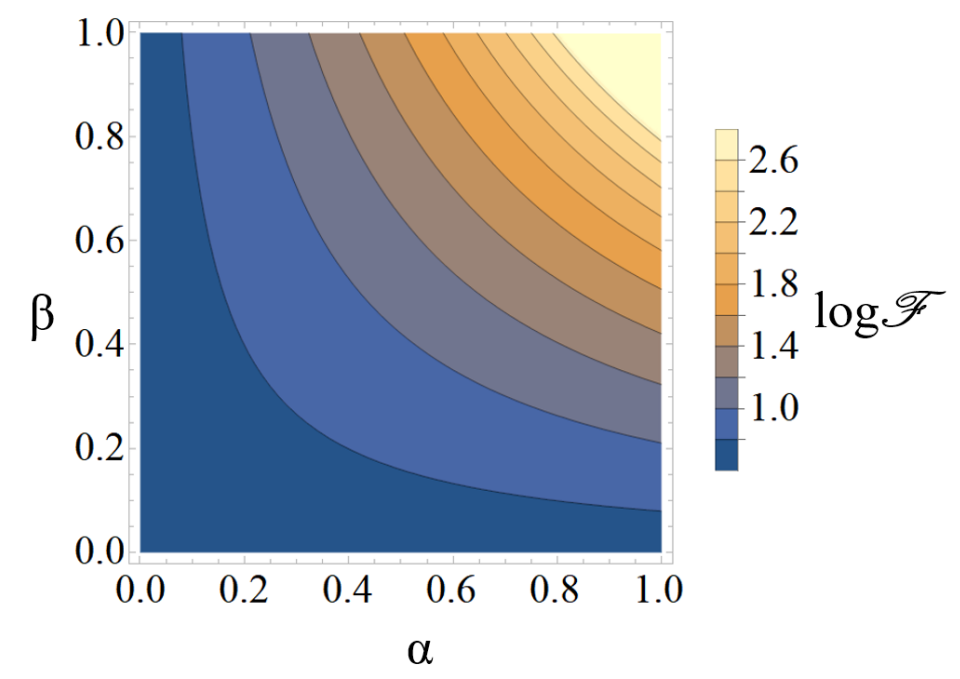

Figure 6. Effect of transmission $(\beta)$ and propagation $(\alpha)$ on the quality factor $Q$, represented by contours of $\log \mathcal{F}$ (Equations (19) and (25)).

Using the general formulation developed here and discussed in the preceding paragraphs, the specific case of RCSS microresonators [27] is now considered. The system in Figure 1, with geometrical dimensions and operational details (shown in Figure 7 (left)) is examined by numerical analysis. To enable a comparison with previous publications $[27,28,36]$, the width of the input waveguide and the microresonator are kept at $0.35 \mu \mathrm{m}$, the gap between the microresonator and the input waveguide is $0.2 \mu \mathrm{m}$, the refractive index of the microresonator and input waveguide is 3.5 , and the total optical path length of the microresonator is $400 \mu \mathrm{m}$ (see Figure 7 (left)). The simulation utilizes the electromagnetic frequency domain (EMFD) analysis, which uses the Finite Element Analysis (FEA) to solve the frequency domain form of Maxwell's equations [37].

A hybrid 2D fine meshing technique (a combination of structured and unstructured) is applied, which generates 733,920 domain elements and 30,429 boundary elements in the entire $(150 \mu \mathrm{m} \times 150 \mu \mathrm{m})$ computational domain (see Figure 7 (right)).

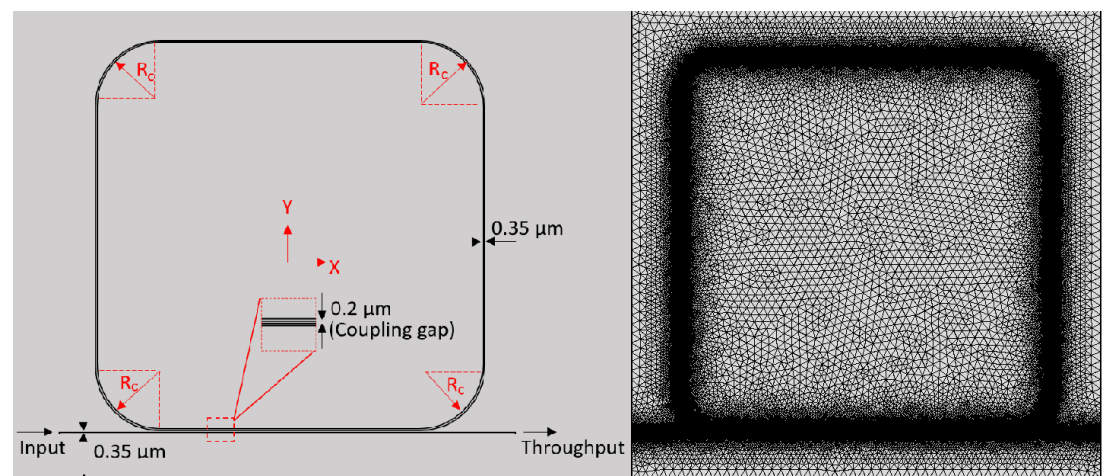

Figure 7. RCSS $R_{c}=15 \mu \mathrm{m}$; (left) Schematic with dimensions; (right) Hybrid meshing for FEA analysis. 
The maximum element size in each domain is equal to $\lambda / 5 \sqrt{n}$, where $\lambda$ is the wavelength and $n$ is the refractive index of the particular domain. The boundary condition at the inlet has a $1 \mathrm{~V} / \mathrm{m}$ electric field in the $z$-direction $\left(E_{z}=1\right.$, TE Mode). The scattering boundary condition that makes the boundaries transparent to a scattered wave and an incoming plane wave is used everywhere else [37].

Multiple cases are simulated using 64-bit windows-based Intel Xeon dual CPUs with 3.20 GHz speed and 8 cores each that share 144 GB RAM. On average, each case was simulated for approximately $18 \mathrm{~h}$ and $25 \mathrm{~min}$, and the spectral response was numerically generated.

\subsection{Effect of the Radius of Curvature in RCSS}

From the results of the numerical simulation, the optical coupling parameters $\alpha$ and $\beta$ of the coupling model presented in this study can be computed. For the specific case of RCSS microresonators [27], the computed values of $\alpha$ and $\beta$ as well as $\epsilon$ and $Q$ are shown in Figure 8. As the radius of curvature at the corners is increased, with the total optical path length kept constant at $400 \mu \mathrm{m}$, the propagation $\alpha$ value changes significantly, ranging between 0.07 and 0.98. Propagation losses are a cumulative effect of bend losses, transition losses, and intrinsic transmission losses, which fluctuate with the changing radius of the bends, even when the total optical path length remains the same. The quality factor $Q$ also varies considerably. The transmission $\beta$ varies less significantly and stays between 0.83 and 0.99. Due to the constant optical path length, the free spectral range remained the same $(\sim 1.1 \mathrm{~nm} \pm 0.1 \mathrm{~nm})$ for all cases presented in this study.

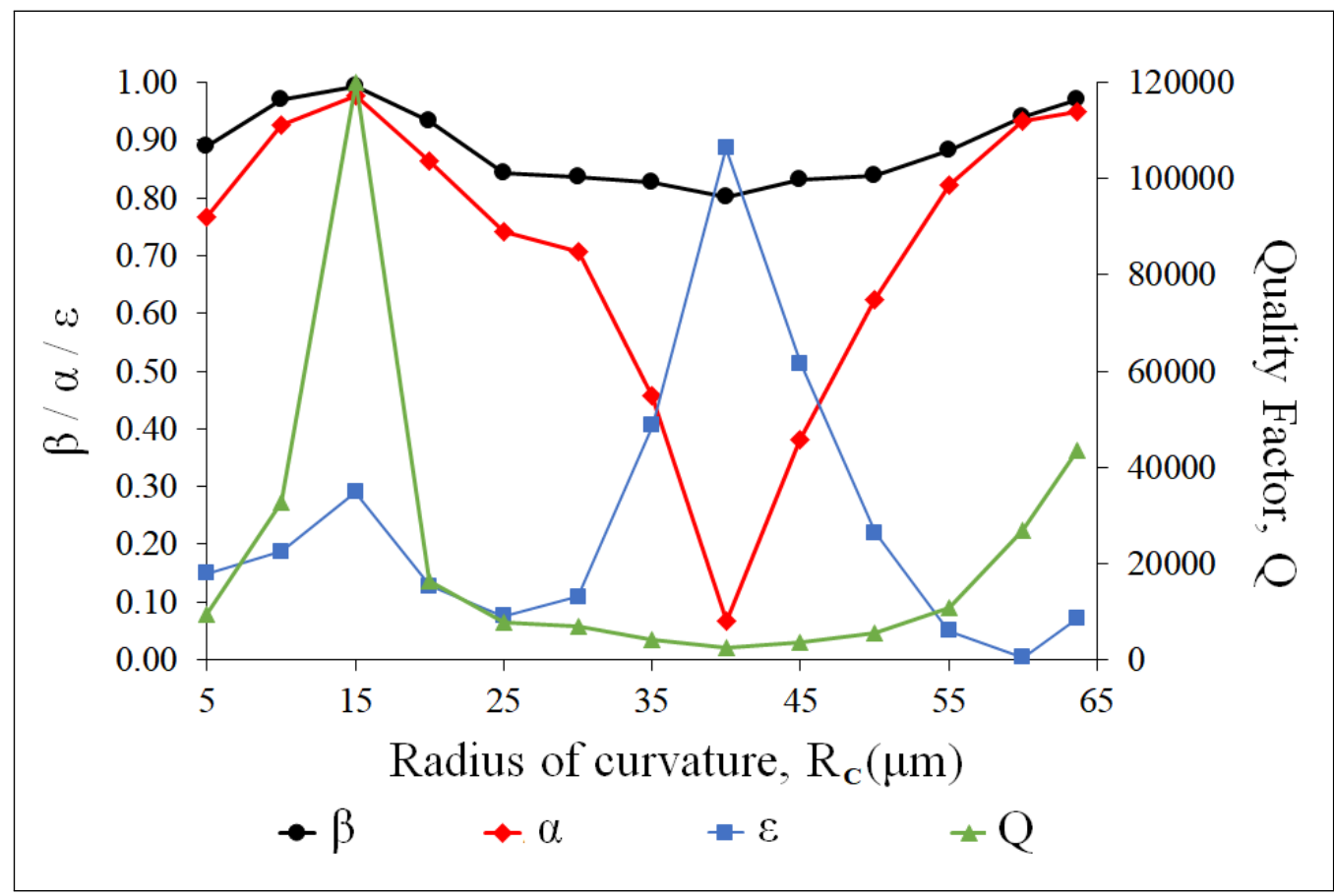

Figure 8. Optical parameters for a waveguide coupled with RCSS microresonator, TE mode, the total optical path length is $400 \mu \mathrm{m}$.

The optimal radius of curvature $R_{c}$ of the RCSS of Panindre and Kumar [27] is $15 \mu \mathrm{m}$, where the quality factor $\left(\sim 10^{5}\right)$ is the highest (the resonance peak is the narrowest) and the throughput port transmissivity shows a very significant dip at resonance (i.e., $Q$ is the highest, $\epsilon$ is low, and the extinction ratio is high). These spectral and quality characteristics are desirable in bio-sensing and optical switching applications. This is because both $\alpha$ and $\beta$ are high in value (close to unity) and the difference between them is small, allowing the resonance signal at the throughput port to have both high quality (narrow linewidth, $\left.\Delta \lambda_{F W H M}\right)$ and a significant transmissivity dip $\left(r_{e} \sim 6 \mathrm{~dB}\right)$. In a similar manner, the least 
desirable radius of curvature is $40 \mu \mathrm{m}$, where the extremely small value of $\alpha$ coupled with the lowest value of $\beta$ leads to a negligible transmissivity dip $\left(r_{e} \sim 0.2 \mathrm{~dB}\right)$ and an extremely small quality factor $\left(\sim 10^{3}\right)$, making the resonance spectral signal indistinguishable from non-resonance regions.

Figure 9 shows the accuracy of the two-parameter transmissivity equation, Equations (10) and (12), with respect to the full spectral signal simulated by the EMFD and the optical experiments for both an RCSS and a ring microresonator. It can be seen that the results from the coupling model and from the EMFD FEA numerical simulation results are virtually identical over the entire free spectral range. These results are also validated by the experimental data, as shown in the figure. Since this is the most important spectral range for applications of resonant microstructures, the coupling model is powerful in describing the optical resonance response. Results from the theoretical model, FEA analysis, and experiments confirm that the round-cornered square-shaped microresonator at an optimum radius of curvature $(\sim 15 \mu \mathrm{m})$ provides a quality factor $\left(\sim 10^{5}\right)$ that is better than that of the conventional microring resonator $\left(\sim 10^{4}\right)$, which is highly desirable for biosensing and other similar applications. Note that the extinction ratio of the conventional ring is $13 \mathrm{~dB}$ and that of the RCSS is $6 \mathrm{~dB}$.

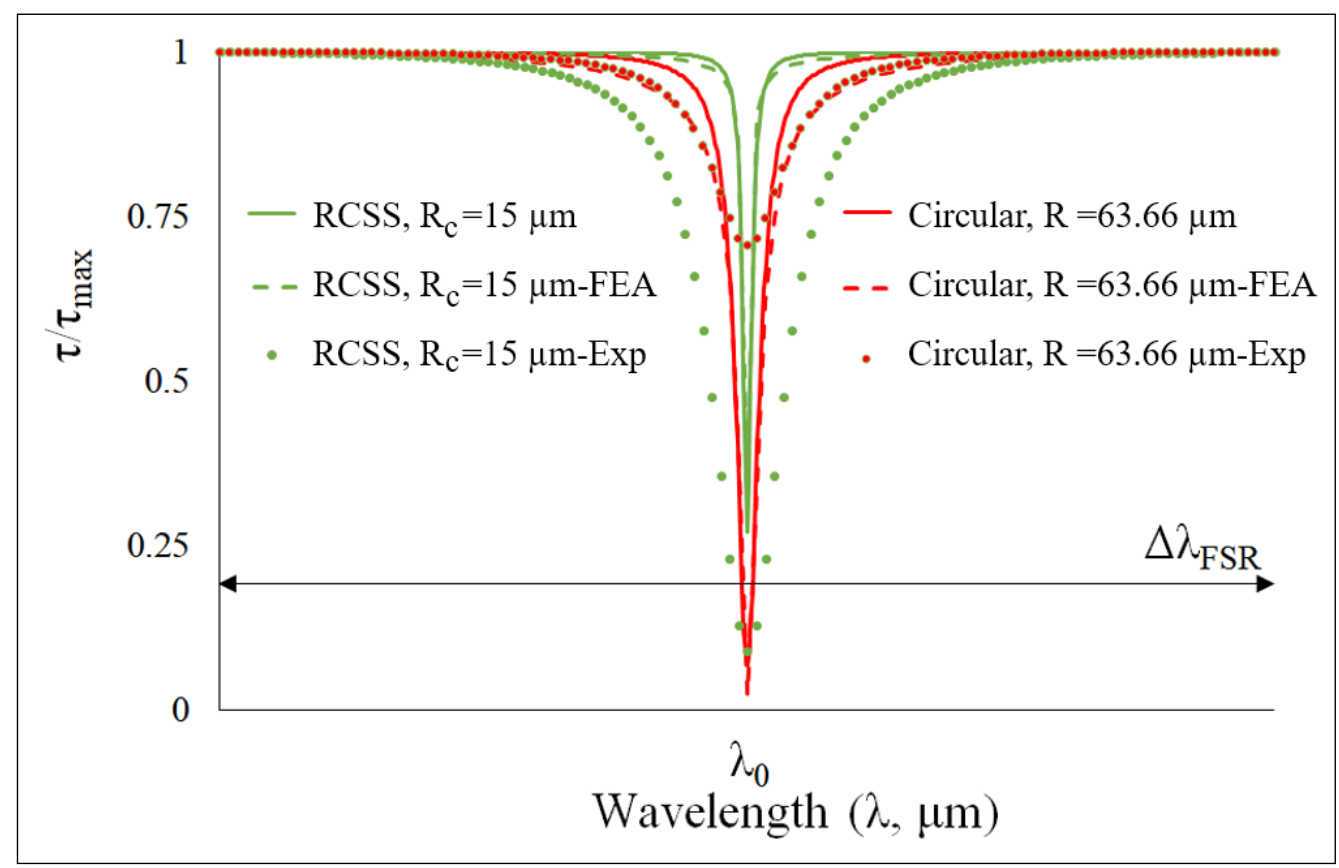

Figure 9. Comparison between the results obtained from the theoretical modeling, the FEA method, and optical experiments for (i) the RCSS microresonator with the optimal radius of curvature $R_{c}=15 \mu \mathrm{m}$ (green) and (ii) the circular microring resonator (radius $63.66 \mu \mathrm{m}$ ), keeping the optical path length at $400 \mu \mathrm{m}$ (red), TE mode.

\subsection{Effect of Rotation in RCSS}

Rotating the RCSS microresonator in the coupling region can remove the restrictions imposed by the requirement of a minimal phase mismatch between the resonator mode and the waveguide mode in these resonator systems, as well as its dependency on the interaction length between the resonator and feed waveguide. The preliminary results of Panindre et al. [36] showed that the quality factor of these resonators can be improved by increasing the rotation angle $\left(0-45^{\circ}\right)$ of the resonator with respect to the feed waveguide around the coupling point. The analysis also established an approach for achieving different quality factors with the same microresonator just by changing its orientation with respect to the feed waveguide; in some cases, the quality factor was significant.

All RCSS microresonator cases discussed in Section 4.2 and Figure 8 have now been rotated by 45 degrees with respect to the feed waveguide. The corresponding values of $\alpha$ 
and $\beta$ as well as $\epsilon$ and $Q$ are computed and plotted, as shown in Figure 10. Comparing Figures 8-10, it can be observed that both $\alpha$ and $\beta$ are close to unity in all cases at a 45-degree rotation, with a radius from $R_{c}=5 \mu \mathrm{m}$ to $55 \mu \mathrm{m}$; in contrast, in the case of no-rotation, the values of $\alpha$ and $\beta$ varied significantly between zero and unity. This results in a higher quality factor for the rotated RCSS at all curvature radii from 0 to $55 \mu \mathrm{m}\left(\sim 10^{5}\right)$ and a consistent lower transmission $\operatorname{dip}$ ( $\epsilon$ between 0 and 0.5 , or $r_{e} \sim 5 \mathrm{~dB}$ to $13 \mathrm{~dB}$ ). However, as the radius of curvature further increases to $60 \mu \mathrm{m}$ and $63.66 \mu \mathrm{m}$ (circular ring), the $\alpha$ and $\beta$ slightly decrease due to an increase in transmission and propagation losses at every point along the circumference of the resonator, leading to a decrease in the quality factor by an order of magnitude (from $\sim 10^{5}$ to $\sim 10^{4}$ ). As the radius of curvature increases, the length of the straight portions of the RCSS microresonator decreases, eventually transforming it into a microring resonator. The straight portions of the RCSS microresonator minimize the transmission and propagation losses but introduce the dependency on the interaction length between the waveguide and the resonator. By rotating the RCSS microresonator by $45^{\circ}$ around the coupling point, this dependency and risk of phase mismatch are eliminated (similar to a circular ring). Therefore, the advantage of minimizing losses due to linear wave propagation along the straight sides is retained, which results in a better quality factor $\left(\sim 10^{5}\right)$ in comparison to conventional microring resonators $\left(\sim 10^{4}\right)$.

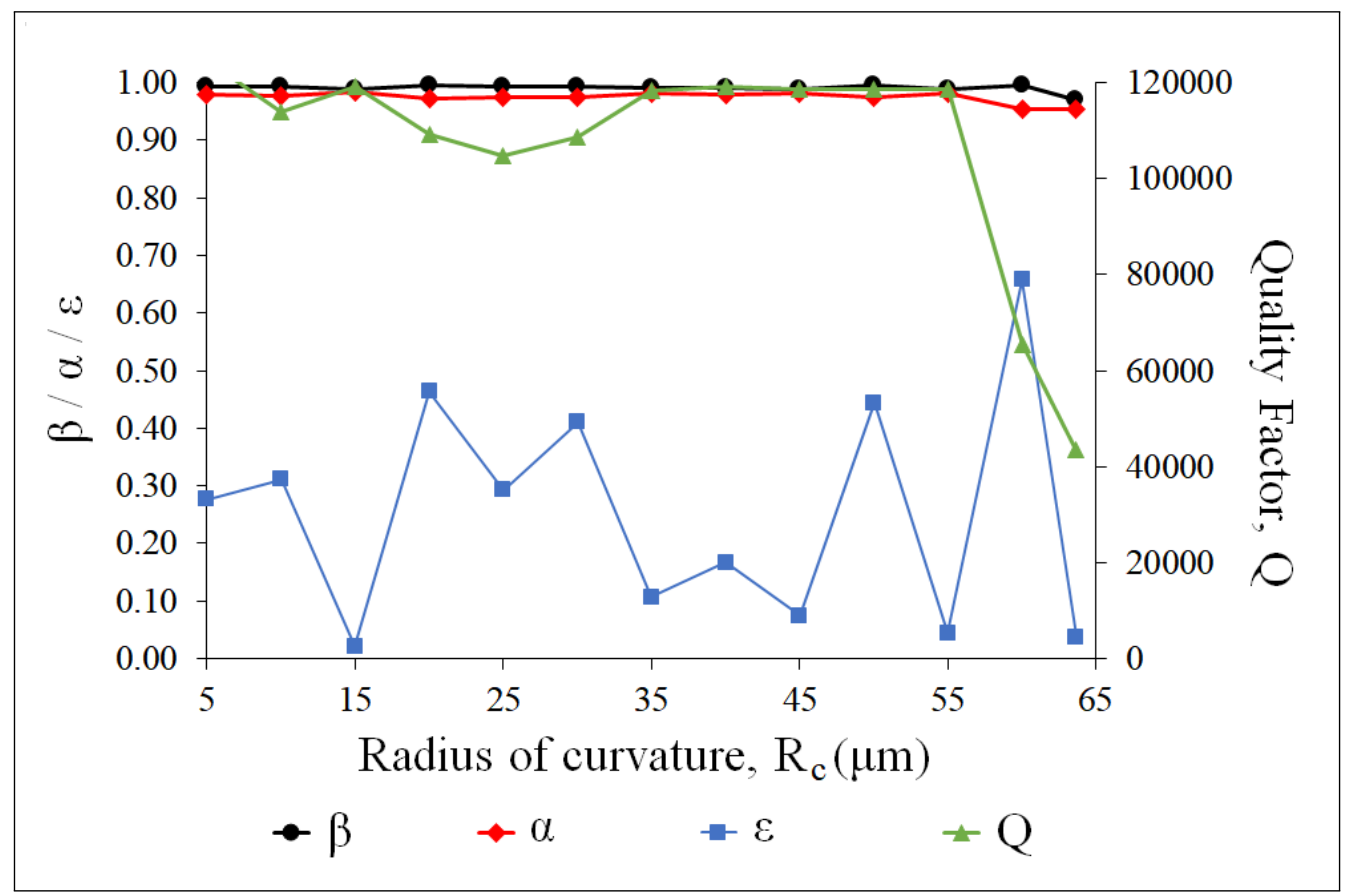

Figure 10. Optical parameters for the rotated RCSS microresonators of the varying radius of curvature $R_{c}$. The rotation angle is $45^{\circ}$ with respect to the feed waveguide around the coupling point. The coupling region is in a central corner of the RCSS.

Additionally, the computed values of $\alpha, \beta, \epsilon$, and $Q$ for the RCSS microresonator $\left(R_{c}=50 \mu \mathrm{m}\right)$, with varying angles of rotation $\theta$ in relation to the feed waveguide around the coupling point are plotted in Figure 11. When the RCSS is rotated from 0 to 45 degrees, the coupling region changes from straight to curved, and mixed in between. Consistent with the findings [36], as $\theta$ increases from 0 to 45 degrees and the dependency on the interaction length between the RCSS resonator and the feed waveguide decreases, both $\alpha$ and $\beta$ steadily increase to reach a value close to unity, with the difference between them continuously decreasing, thus leading to a consistent and significant improvement in the quality factor, which underscores the importance of coupling.

By comparing the results of Figures 8 and 10, it can be seen that the same microresonators exhibit different optical characteristics when rotated, i.e., the values of the quality 
factor and the extinction ratio vary significantly. Rotating changes the coupling characteristics, which demonstrates the importance of coupling to the optical performance. This phenomenon is further investigated for the RCSS microresonator with a radius of curvature of $R_{c}=50 \mu \mathrm{m}$ with FEA analysis (see Figure 12), whose results have been validated with the data from the optical experiments. The results of both the FEA and the optical experiments demonstrate a significant improvement in the quality factor (from $\sim 10^{3}$ to $\sim 10^{5}$ ) and transmission dip ( $r_{e}$ increases from $6 \mathrm{~dB}$ to $8.2 \mathrm{~dB}$ ) at resonance, with the rotation of the RCSS resonator $\left(R_{c}=50 \mu \mathrm{m}\right)$ around the coupling point. This finding can be envisioned in Figure 13, noting that the corresponding values of the quality factor for the experimental results are lower than those for the FEA analysis due to several experimental and fabrication limitations. For instance, the coupling gap and width of the waveguide and microresonator inputs have experimental uncertainties (a sigma variation of $3.84 \mathrm{~nm}$ in width and $1.38 \mathrm{~nm}$ in thickness), and may not be the same as the dimensions used for the FEA and theoretical analyses. Additionally, optical microresonators are extremely sensitive to the thermal environment during the optical experiment, both due to the temperature-dependent refractive index of the microresonator and the bulk refractive index of the surrounding medium $[7,8,16]$. Other experimental uncertainties are the roughness of microresonator surfaces and attenuation/noise at input and output connections. However, the trend of the experimental results on the effect of rotation around the coupling point in RCSS microresonators is in a good agreement with the FEA analysis.

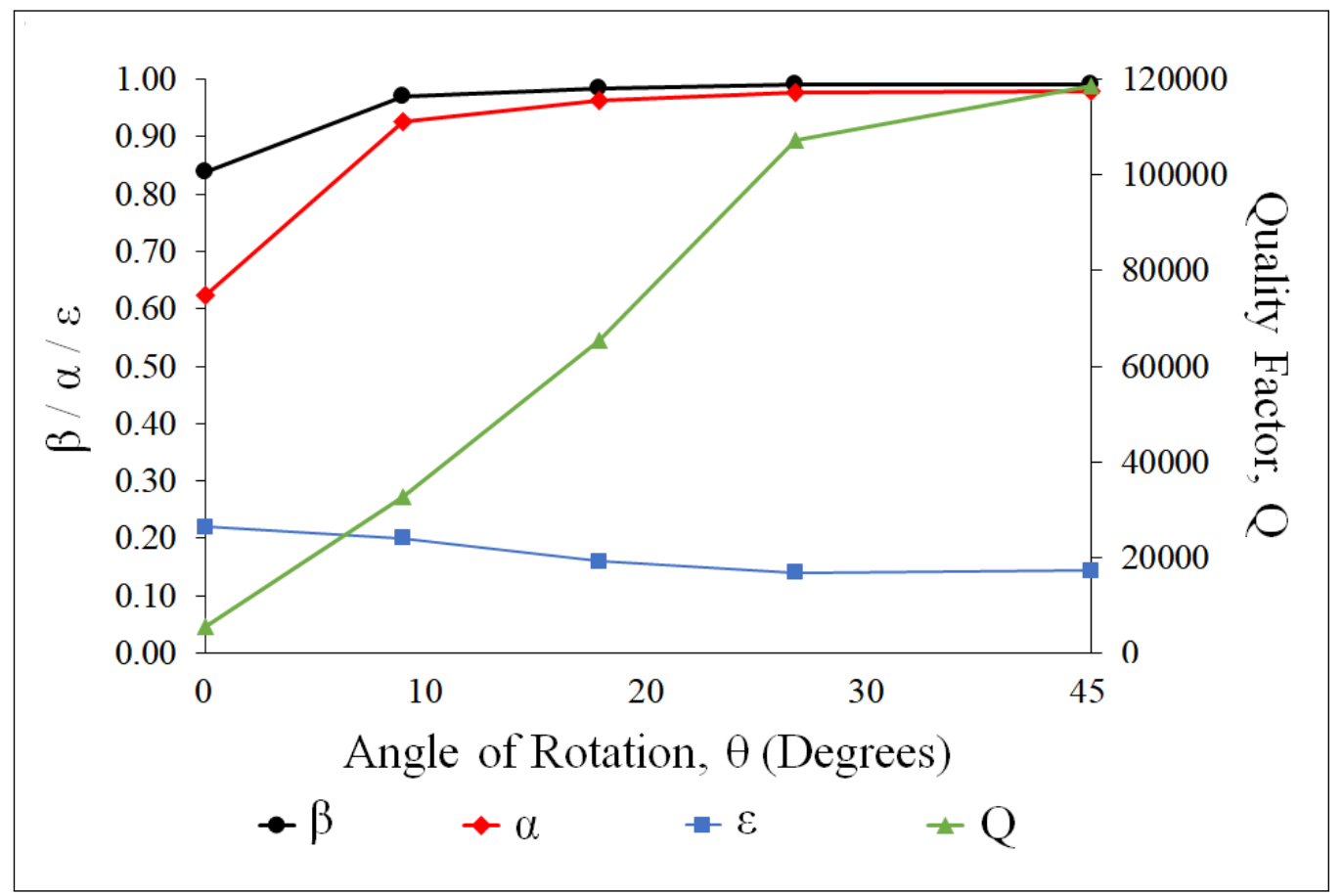

Figure 11. Optical parameters for an RCSS microresonator $\left(R_{c}=50 \mu \mathrm{m}\right)$ with varying angles of rotation in relation to the feed waveguide around the coupling point.

Panindre and Kumar [27] showed that by changing the radius of curvature of the round-cornered square-shaped (RCSS) microresonator, its quality factor can be varied, and the quality factor can be higher than that of the circular ring microresonator at optimum radius of curvature. The current study establishes that the RCSS microresonator of lower quality [27] can be improved by its rotation to a quality factor better than that of a circular ring. This significant result firmly establishes the RCSS, and by extension other geometries with straight sides and curved corners (e.g., polygons, racetrack), as competitive alternatives to conventional circular microresonators. 


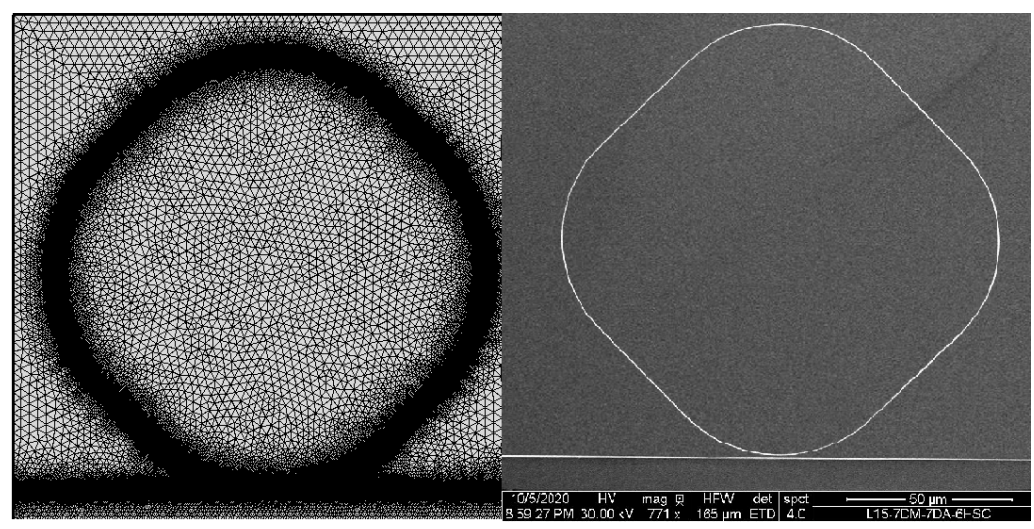

Figure 12. RCSS $\left(R_{c}=50 \mu \mathrm{m}\right)$ with $45^{\circ}$ rotation in relation to the feed waveguide around the coupling point. (left) Hybrid meshing of the RCSS for FEA analysis; (right) SEM image of the fabricated RCSS for optical experiments.

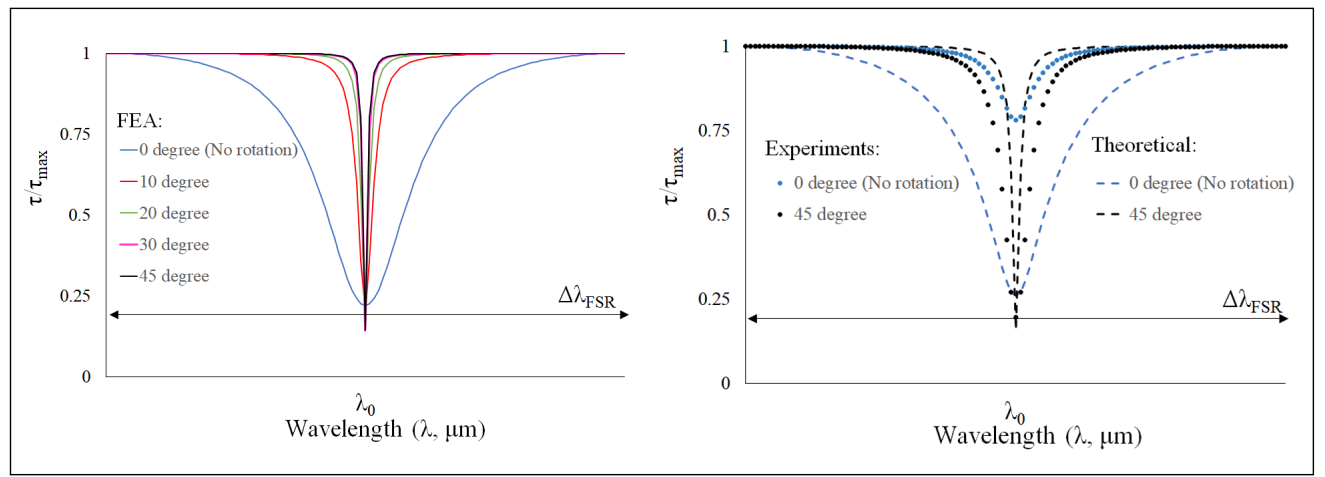

Figure 13. Comparison of the optical response of the on-chip RCSS device with different angles of rotations: (left) FEA method, (right) optical experiments, and theoretical modeling. Here, $R_{c}=50 \mu \mathrm{m}$, and the total optical path length is $400 \mu \mathrm{m}$, TE mode.

\section{Conclusions}

We applied a coupling model to an on-chip structure consisting of a round-cornered square-shaped (RCSS) resonator as an optical filter. The theoretical relationships established in this study indicate that in addition to intrinsic losses in the resonator, the characteristics of the coupling also significantly influence the resonant spectral response of the coupled waveguide-resonator system. The quality factor and finesse of the microresonators were shown to be functions of both transmission $(\beta)$ and resonator propagation $(\alpha)$ values. Theoretical results provided a good understanding of the influencing parameters, and a simple expression that uses only $\alpha$ and $\beta$ to represent the entire spectral response was demonstrated.

It was seen that in order to obtain a high-quality factor and the greatest transmission signal dip at resonance, $\alpha$ and $\beta$ should be similar in value and as close to unity as possible. Furthermore, these approaches were used to confirm that the quality factor and transmission dip at resonance improve significantly with the rotation of the RCSS resonator in relation to the waveguide around the coupling point. Additionally, the study also experimentally, theoretically, and numerically validated that the RCSS microresonator, at an optimum radius of curvature and an optimum angle with respect to the input waveguide, provides a significantly better resonance response than the conventional microring resonator. The present analysis can assist in improving the coupling characteristics and optical performance of resonators of polygonal, circular, or any other geometries.

The present study establishes that round-cornered square-shaped (RCSS) microresonators can be competitive alternatives to circular ring microresonators, and that with the proper selection of the radius of curvature and the angle of rotation with respect to the 
input waveguide, the RCSS can deliver a better quality factor as compared to a circular ring microresonator.

Author Contributions: Conceptualization, P.P. and S.K.; investigation, B.P.; methodology, P.P. and N.S.S.M.; supervision, S.K.; validation, B.P. and M.R.; visualization, N.S.S.M.; writing-original draft, P.P. and N.S.S.M.; writing-review and editing, P.P., N.S.S.M., B.P., M.R. and S.K. All authors have read and agreed to the published version of the manuscript.

Funding: This research was partially funded by the research grant, ADEC Award for Research Excellence (AARE-0073) from the Abu Dhabi Education Council.

Institutional Review Board Statement: Not applicable.

Informed Consent Statement: Not applicable.

Data Availability Statement: The data that support the findings of this study are available from the corresponding author upon reasonable request.

Conflicts of Interest: The authors declare no conflict of interest.

$\begin{array}{ll}\text { Abbreviations } \\ \text { RCSS } & \text { Round-cornered square-shaped } \\ \text { FWHM } & \text { Full-width at half-maximum } \\ \text { EMFD } & \text { Electromagnetic finite domain } \\ \text { FEA } & \text { Finite element analysis } \\ \text { RI } & \text { Refractive index } \\ \text { FSR } & \text { Free spectral range }\end{array}$

\section{References}

1. Hamzah, H.; Lees, J.; Porch, A. Split ring resonator with optimised sensitivity for microfluidic sensing. Sens. Actuators A Phys. 2018, 276, 1-10. [CrossRef]

2. Rabus, D.G. Integrated Ring Resonators: The Compendium; Springer Series in Optical Sciences; Springer: Berlin/Heidelberg, Germany, 2007. Available online: https:/ / www.springer.com/de/book/9783540687863 (accessed on 27 November 2020).

3. Wei, X.; Panindre, P.; Zhang, Q.; Song, Y.-A. Increasing the detection sensitivity for dna-morpholino hybridization in subnanomolar regime by enhancing the surface ion conductance of pedot:pss membrane in a microchannel. ACS Sens. 2016, 1, 862-865. [CrossRef]

4. Krasnokutska, I.; Tambasco, J.-L.J.; Peruzzo, A. Tunable large free spectral range microring resonators in lithium niobate on insulator. Sci. Rep. 2019, 9, 11086. [CrossRef] [PubMed]

5. Steglich, P.; Hülsemann, M.; Dietzel, B.; Mai, A. Optical Biosensors Based on Silicon-On-Insulator Ring Resonators: A Review. Molecules 2019, 24, 519. [CrossRef]

6. Henriksson, A.; Kasper, L.; Jäger, M.; Neubauer, P.; Birkholz, M. An approach to ring resonator biosensing assisted by dielectrophoresis: Design, simulation and fabrication. Micromachines 2020, 11, 9543. [CrossRef]

7. Panindre, P. Label-Free Optical Sensing of a Single Exosome in a Microfluidic Chip. Ph.D. Thesis, Polytechnic Institute of New York University, Brooklyn, NY, USA, 2016.

8. Mousavi, N.S.S.; Panindre, P.; Kumar, S. Design Optimization of a Single-Mode Microring Resonator for Label-Free Detection of Biomarkers within a Tunable Spectral Range of $2 \mathrm{~nm}$. In Biomedical Imaging and Sensing Conference; Matoba, O., Awatsuji, Y., Luo, Y., Yatagai, T., Aizu, Y., Eds.; SPIE: Yokohama, Japan, 2018. [CrossRef]

9. Puckett, M.W.; Liu, K.; Chauhan, N.; Zhao, Q.; Jin, N.; Cheng, H.; Wu, J. 422 Million Intrinsic Quality Factor Planar Integrated All-Waveguide Resonator with Sub-MHz Linewidth. Nat. Commun. 2021, 12, 934. [CrossRef]

10. Spencer, D.T.; Bauters, J.F.; Heck, M.J.R.; Bowers, J.E. Integrated Waveguide Coupled $\mathrm{Si}_{3} \mathrm{~N}_{4}$ Resonators in the Ultrahigh-Q Regime. Optica 2014, 1, 153. [CrossRef]

11. Brunetti, G.; Olio, F.D.; Conteduca, D.; Armenise, M.N.; Ciminelli, C. Comprehensive Mathematical Modelling of Ultra-High Q Grating-Assisted Ring Resonators. J. Opt. 2020, 22, 035802. [CrossRef]

12. Yariv, A. Critical coupling and its control in optical waveguide-ring resonator systems. IEEE Photon. Technol. Lett. 2002, 14, 483-485. [CrossRef]

13. Heebner, J.; Wong, V.; Schweinsberg, A.; Boyd, R.; Jackson, D. Optical transmission characteristics of fiber ring resonators. IEEE J. Quantum Electron. 2004, 40, 726-730. [CrossRef]

14. Timotijevic, B.; Gardes, F.; Headley, W.; Reed, G.; Paniccia, M.; Cohen, O.; Hak, D.; Masanovic, G. Multi-stage racetrack resonator filters in silicon-on-insulator. J. Opt. A Pure Appl. Opt. 2006, 8, S473. [CrossRef] 
15. Bogaerts, W.; Heyn, P.D.; Vaerenbergh, T.V.; Vos, K.D.; Selvaraja, S.K.; Claes, T.; Dumon, P.; Bienstman, P.; Thourhout, D.V.; Baets, R. Silicon microring resonators. Laser Photon. Rev. 2012, 6, 47-73. [CrossRef]

16. Panindre, P.; Kumar, S. Temperature effects on optical resonances in single-mode circular ring and squircular resonators. In Proceedings of the ASME 2017 Heat Transfer Summer Conference, Bellevue, WA, USA, 9-12 July 2017; Volume 2, p. V002T15A001. [CrossRef]

17. Chen, G.; Jiang, C. Reverse design of microring resonator channel dropping filters. Results Phys. 2020, 19, 103380. [CrossRef]

18. Kargar, A.; Lee, C. Analysis of racetrack resonators in surface sensing applications. In Proceedings of the PhotonicsGlobal@Singapore, Singapore, 8-11 December 2008; pp. 1-4. [CrossRef]

19. Chin, M.; Ho, S. Design and modeling of waveguide-coupled single-mode microring resonators. J. Light. Technol. 1998, 16, 1433. [CrossRef]

20. Soltani, M.; Yegnanarayanan, S.; Li, Q.; Adibi, A. Systematic engineering of waveguide-resonator coupling for silicon microring/microdisk/racetrack resonators: Theory and experiment. IEEE J. Quantum Electron. 2010, 46, 1158-1169. [CrossRef]

21. Poon, A.; Courvoisier, F.; Chang, R.K. Multimode resonances in square-shaped optical microcavities. Opt. Lett. 2001, 26, 632-634. [CrossRef] [PubMed]

22. Latiff, A.A.; Rahim, A.; Rafis, H.; Jaafar, A.; Gannapathy, V.R.; Zainuddin, M.N.S. Design high-q square resonator add-drop filter for cwdm application. Aust. J. Basic Appl. Sci. 2013, 7, 364-367. Available online: http://www.ajbasweb.com/old/ajbas/2013 /August/364-367.pdf (accessed on 21 September 2020).

23. Moon, H.-J.; Sun, S.-P.; Park, G.-W.; Lee, J.-H.; An, K. Whispering gallery mode lasing in a gain-coated square microcavity with round corners. Jpn. J. Appl. Phys. 2003, 42, L652. [CrossRef]

24. Boriskina, S.V.; Benson, T.M.; Sewell, P.; Nosich, A.I. Optical modes in 2-d imperfect square and triangular microcavities. IEEE J. Quantum Electron. 2005, 41, 857-862. [CrossRef]

25. Guo, W.-H.; Huang, Y.-Z.; Lu, Q.-Y.; Yu, L.-J. Modes in square resonators. IEEE J. Quantum Electron. 2003, 39, 1563-1566. [CrossRef]

26. Fong, C.Y.; Poon, A.W. Mode field patterns and preferential mode coupling in planar waveguide-coupled square microcavities. Opt. Express 2003, 11, 2897-2904. [CrossRef]

27. Panindre, P.; Kumar, S. Effect of rounding corners on optical resonances in single-mode sharp-cornered microresonators. Opt. Lett. 2016, 41, 878-881. [CrossRef] [PubMed]

28. Panindre, P.; Kumar, S. Closed Loop Microresonators Having Linear Portions and Filleted Corners, Systems Including Such Microresonators, and Methods of Fabricating Such Microresonators. U.S. Patent 10,684,418, 7 November 2019. (Pub. No. US 2019/0339455 A1). Available online: https:/ / www.freepatentsonline.com/y2019/0339455.html (accessed on 14 November 2020).

29. Marcatili, E.A.J. Dielectric Rectangular Waveguide and Directional Coupler for Integrated Optics. Bell Syst. Tech. J. 1969, 48, 2071-2102. [CrossRef]

30. Xu, Y.; Li, Y.; Lee, R.K.; Yariv, A. Scattering-Theory Analysis of Waveguide-Resonator Coupling. Phys. Rev. E 2000, 62, 7389-7404. [CrossRef] [PubMed]

31. Long, Y.; Wang, J. Optically-Controlled Extinction Ratio and Q-Factor Tunable Silicon Microring Resonators Based on Optical Forces. Sci. Rep. 2015, 4, 5409. [CrossRef]

32. Sumetsky, M. Optimization of Optical Ring Resonator Devices for Sensing Applications. Opt. Lett. 2007, 32, 2577. [CrossRef]

33. Lien, S. Chuang, Physics of Photonic Devices, 2nd ed.; John Wiley \& Sons: Hoboken, NJ, USA, 2012.

34. Geuzebroek, D.H.; Driessen, A. Ring-Resonator-Based Wavelength Filters; Springer: Berlin/Heidelberg, Germany, 2006; Volume 123, pp. 341-379. Available online: http:/ /link.springer.com/10.1007/3-540-31770-8_9 (accessed on 22 September 2020).

35. Applied Nanotools Inc.: X-ray Optics and Integrated Photonics. Available online: https://www.appliednt.com/ (accessed on 18 August 2020).

36. Panindre, P.; Mousavi, N.S.S.; Kumar, S. Effect of rotation on quality factor of single-mode optical resonances in roundcornered square-shaped resonators. In Physics and Simulation of Optoelectronic Devices XXVI; SPIE: San Francisco, CA, USA, 2018; Volume 10526, p. 1052627. [CrossRef]

37. COMSOL. Comsol Multiphysics User Guide (Version 5.2 a); COMSOL Multiphysics, AB: Stockholm, Sweden, 2016. 